\title{
Temporal Heterogeneous Information Network Embedding
}

\author{
Hong Huang ${ }^{1,2,3 *}$, Ruize Shi ${ }^{1,2,3}$, Wei Zhou ${ }^{3}$, Xiao Wang ${ }^{4 *}$, Hai Jin ${ }^{1,2,3}$ and Xiaoming Fu ${ }^{5}$ \\ ${ }^{1}$ National Engineering Research Center for Big Data Technology and System \\ ${ }^{2}$ Service Computing Technology and Systems Laboratory \\ ${ }^{3}$ Huazhong University of Science and Technology, China \\ ${ }^{4}$ Beijing University of Posts and Telecommunications, China \\ ${ }^{5}$ University of Goettingen, Germany \\ \{honghuang, rzshi, zhouw, hjin\}@hust.edu.cn, xiaowang@bupt.edu.cn,fu@cs.uni-goettingen.de
}

\begin{abstract}
Heterogeneous information network (HIN) embedding, learning the low-dimensional representation of multi-type nodes, has been applied widely and achieved excellent performance. However, most of the previous works focus more on static HINs or learning node embeddings within specific snapshots, and seldom attention has been paid to the whole evolution process and capturing all dynamics. In order to fill the gap of obtaining multitype node embeddings by considering all temporal dynamics during the evolution, we propose a novel temporal HIN embedding method (THINE). THINE not only uses attention mechanism and meta-path to preserve structures and semantics in HIN but also combines the Hawkes process to simulate the evolution of the temporal network. Our extensive evaluations with various real-world temporal HINs demonstrate that THINE achieves the SOTA performance in both static and dynamic tasks, including node classification, link prediction, and temporal link recommendation.
\end{abstract}

\section{Introduction}

In recent years, network embedding has attracted more and more attention on account of its outstanding performance. It maps nodes to a low-dimensional space and preserves the features and structures of the network simultaneously. A lot of excellent algorithms such as Deepwalk [Perozzi et al., 2014], LINE [Tang et al., 2015b] have been successfully applied in various network-related tasks, such as node classification, node clustering, and link prediction.

However, these methods all focus on homogeneous networks, while most real-world data are heterogeneous information networks (HINs) with multiple types of nodes and relations. For example, an academic network generally has three types of nodes: Author (A), Paper (P), Conference (C); and multiple types of relations: co-author relations, reference relations, write/written relations between authors and papers, publish/published relations between papers and conferences.

\footnotetext{
${ }^{*}$ Corresponding Author
}

In HIN, different types of nodes and edges could generate various embeddings and contain more complex structures and interrelations. Therefore, more and more researchers have paid attention to HINs such as PTE [Tang et al., 2015a], Metapath2Vec [Dong et al., 2017], and MAGNN [Fu et al., 2020].

Nonetheless, most of the current works are proposed for static HIN, which in contrast to the reality, where the HIN actually evolves over time. Examples include academic networks where authors may publish different papers in different years, and the grade of business varies according to users' reviews with time changing in Yelp. Therefore, simply treating a temporal HIN as a static HIN will inevitably not accurately capture the structure and semantics when HIN changes.

As a result, there is a growing need to understand temporal HIN. However, it faces two serious challenges. First, how to effectively preserve the structural and semantic dynamics in temporal HINs? Dynamics describe all the changes of nodes and edges in HINs during its evolution, including the nodes' addition, edges' deletion, etc. Thus, accurately capturing the dynamics is critical to study temporal HIN. However, most previous works like DHNE [Yin et al., 2019], model temporal HINs using snapshots by simply dividing the time into several periods, which would lose dynamics within snapshots.

Another challenge is how to capture the temporal influence between heterogeneous nodes? Unlike homogeneous networks, HINs contain multiple types of nodes and edges, thus preserve more complex semantics and structures. For example, in an academic network, we usually consider the temporal influence from the same types of nodes like authorauthor or paper-paper. Moreover, in HINs, we should also consider the temporal effects from different types of nodes like author-paper. But due to the difficulty of simulating the influence between heterogeneous nodes, the majority of previous works like HDGAN [Li et al., 2020], DyHNE [Wang et al., 2020] only consider the temporal influence from the same types of nodes merely.

To this end, we propose THINE, a novel temporal HIN embedding model for capturing the dynamics among all types of nodes. We first define various meta-paths to capture the semantics and structures of a HIN. Then for a specific downstream task, we generate candidate meta-path sets related to the task. By modeling the temporal influence between nodes with the Hawkes process, we obtain each node's embedding. Moreover, two levels of attention mechanisms are 
applied to distinguish weight in various aspects. One is for different types of meta-path, and the other is for the distance of the neighbor nodes. Experiments on various real-world datasets show that, compared with several SOTA methods, our THINE performs better in both static and dynamic tasks.

The contributions can be summarized as follows:

- We study the problem of temporal HIN embedding by considering the evolutionary dynamics.

- We initiate a novel temporal HIN embedding model, which uses meta-path to capture structural and semantic information of HIN, leverages the Hawkes process to model network evolution, and applies two levels of attention to capture structural and semantic differences respectively.

- Experimental results on three real-world datasets show that THINE outperforms several SOTA methods.

\section{Preliminaries}

The evolution of a HIN can be regarded as the addition and deletion of nodes or edges at different times. Traditionally, we define temporal HIN in the following:

Definition 1: Temporal HIN. A temporal HIN is defined as $\mathcal{G}=(\mathcal{V}, \mathcal{E}, \mathcal{T}, \phi, \varphi)$, where $\mathcal{V}$ indicates the set of nodes, $\mathcal{E}$ indicates the set of edges and $\mathcal{T}$ denotes the set of timestamps. Besides, there are two mapping functions $\phi: \mathcal{V} \rightarrow \mathcal{A}, \varphi:$ $\mathcal{E} \rightarrow \mathcal{R}$, while $\mathcal{A}, \mathcal{R}$ indicate the set of types for nodes and edges. For each HIN, it satisfies that $|\mathcal{A}|+|\mathcal{R}|>2$.

Especially, an edge $e_{i j}^{t} \in \mathcal{E}$ represents a relation established between nodes $v_{i}$ and $v_{j}$ at time $t$. It is worth noting that two nodes may establish multiple relations at different times. For instance, conference $(C)$ may publish multiple papers by the same author (A), so it is probable that there exist numerous relations between $\mathrm{A}$ and $\mathrm{C}$ in academic networks.

Definition 2: Influence of Node Pairs. The influence of node pairs indicates the contribution of two nodes to establish a connection between them. Given a node pair $\left(v_{x}, v_{y}\right)$, where $v_{x}, v_{y} \in \mathcal{V}$ and $\mathcal{V}$ is the set of nodes, the influence of node pair is $\eta_{x, y}$. Formally, we have:

$$
\eta_{x, y}=-\left\|u_{x}-u_{y}\right\|^{2}
$$

where $u_{x}, u_{y}$ represent the embeddings of nodes $v_{x}$ and $v_{y}$.

Definition 3: Meta-path. Given a temporal HIN $\mathcal{G}=(\mathcal{V}, \mathcal{E}, \mathcal{T}, \phi, \varphi)$, meta-path $\mathcal{M}$ is a path which is defined as $a_{1} \stackrel{r_{1}}{\longrightarrow} a_{2} \stackrel{r_{2}}{\longrightarrow} a_{3} \stackrel{r_{3}}{\longrightarrow} \ldots \stackrel{r_{l-1}}{\longrightarrow} a_{l}$, where $r_{i} \in \mathcal{R}$, $a_{i} \in \mathcal{A}$, and $\mathcal{A}, \mathcal{R}$ indicate the set of types for nodes and edges. Obviously, a meta-path describes a complex relation between types $a_{1}$ and $a_{l}$. Especially, a sequence of nodes $\left(v_{1}, v_{2}, \ldots, v_{l}\right)$ that satisfies the law of meta-path $\mathcal{M}$ is a path instance $m$ of meta-path $\mathcal{M}$.

Definition 4: Candidate Meta-path Set. The candidate meta-path set $S(t)$ of a temporal edge $e_{i j}^{t} \in \mathcal{E}$, is a set that includes all the path instances which involve source node $v_{i}$ and are generated before time $t$. Note that a path instance formed before time $t$ means that all of the temporal edges it included generated before time $t$.

In this paper, our goal is to obtain multi-type node embeddings with consideration of temporal dynamics. Formally, we define our problem as follows:
Problem. Temporal HIN Embedding. Given a temporal HIN $\mathcal{G}=(\mathcal{V}, \mathcal{E}, \mathcal{T}, \phi, \varphi)$, the goal is to learn a mapping function $f: \mathcal{V} \rightarrow \mathbb{R}^{d}$, where $d \ll|\mathcal{V}|$ and $d$ represents the number of embedding dimensions. The function $f$ needs to not only capture the temporal dynamics of the network but also consider the influences between all types of nodes.

\section{The Proposed Model}

\subsection{Model Overview}

In this section, we will explain the details of our proposed model THINE, which can capture the structures and semantics of HIN and combine the impact of temporal dynamics simultaneously. As Figure 1 shown, THINE gets the structural interactions between different types of nodes by meta-path based random walk. Then, we obtain the candidate meta-path set for each edge to model the dynamic structures and semantics of temporal HIN with the Hawkes process [Hawkes, 1971]. Furthermore, optimizing with structural-level and semantic-level attention mechanisms that distinguish the impacts of different relations, we aggregate the effects for every node to obtain multi-type node embeddings.

\subsection{THINE Model}

Capturing semantics with meta-path. THINE first uses meta-path based random walk to extract information of HIN. The construction of meta-path determines what semantics and structures we can capture. Therefore, the selection of metapath is critical to study HINs. The key to defining meta-path is to contain as many semantics as possible. For example, for academic networks, besides the mate-path of author-paper relation that considered by previous models, we also consider the meta-path of paper-paper relation, written as APPA. In total, the meta-path we defined are listed in Table 1. With those meta-paths, we can well preserve the semantics in HINs. Moreover, the nodes and edges in the network are influenced by the nodes themselves and the related candidate meta-path set. Hence, based on the influence of node pairs, we model the influence of candidate set to understand temporal HIN.

Modeling dynamics of candidate meta-path set. Furthermore, we model the influence of candidate meta-path set to capture semantics and structures of temporal HIN with the Hawkes process. Generally, the Hawkes process is used to simulate the impact of past events on the present. Obviously, the older events are, the fewer impacts they have on today. Specifically, for THINE, we apply an attention with the Hawkes process for every influence. Therefore, the influence of candidate meta-path set, to wit the influence of all related meta-path instances, is formally defined as

$$
\eta_{s}(t)=\sum_{t_{m}<t} \eta_{m}(t)
$$

where $m$ is one meta-path instance, and $t_{m}<t$ indicates the meta-path instance $m$ generated before time $t$. For convenience, we use $\eta_{i}, i \in(s, m, e)$ to represent the influence of candidate meta-path set, one meta-path, and one temporal edge. Therefore, $\eta_{s}(t), \eta_{m}(t)$, and $\eta_{e}(t)$ indicate corresponding influence before time $t$. So that we need to primarily 


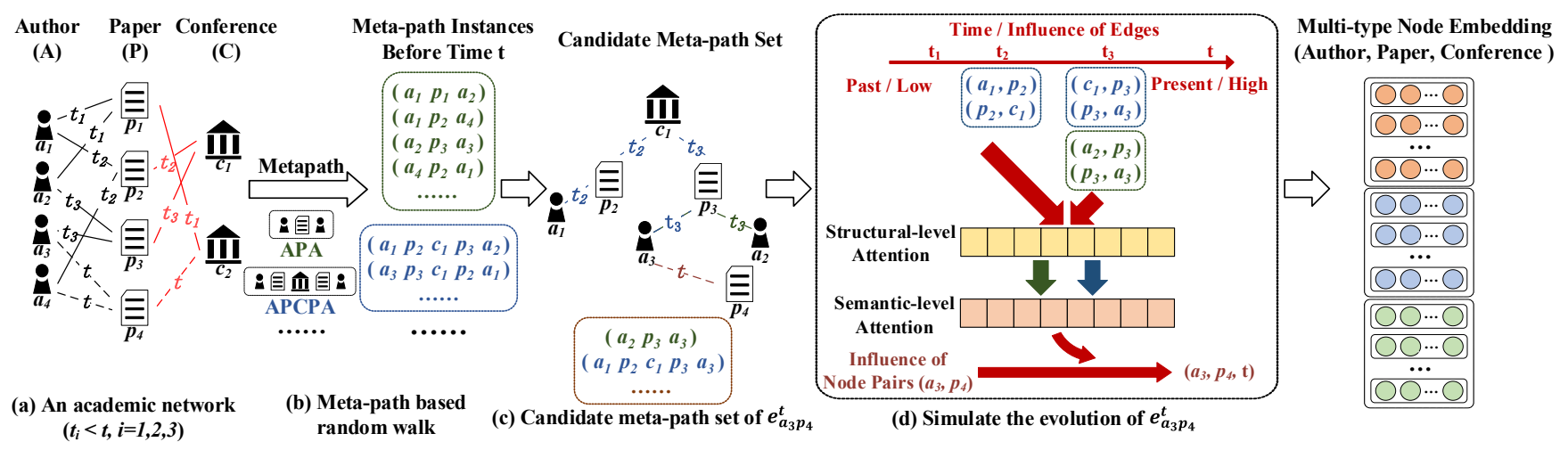

Figure 1: The overall architecture of the proposed THINE

model the influence of one meta-path instance $\eta_{m}(t)$, which can be considered as the influence of all the edges it included. Thus, it is defined as

$$
\eta_{m}(t)=\sum_{e_{i j} \in m} \eta_{e_{i j}}(t)
$$

where $e_{i j}$ represents an edge between nodes $v_{i}$ and $v_{j}$. One edge can be represented by two nodes it connects. So we model the influence of one temporal edge by using the influence of node pairs and the Hawkes process, which means

$$
\eta_{e_{i j}}(t)=\eta_{i, j} \times \sum_{t_{i, j}<t} \kappa\left(t-t_{i, j}\right),
$$

where $t_{i, j}$ represents the timestamp of edge $e_{i j}^{t}, \kappa\left(t-t_{i, j}\right)$ describes the time decay effect and we define $\kappa\left(t-t_{i, j}\right)=$ $e^{\left(-\delta_{i}\left(t-t_{i, j}\right)\right)}$. Moreover, $\delta$ is a trainable parameter related to nodes and adjusts the ratio of time decay effect.

Note that we select a subset of the candidate meta-path set for training due to the computational complexity. Specifically, we choose $n$ meta-path instances generated most recently from the time $t$ while $n$ is a hyperparameter. Likely, for one meta-path instance, we select $z$ candidate edges closest to the source node to train and $z$ is also a hyperparameter.

Optimization with attention mechanism. Specifically, we show how THINE calculates the influence of the candidate meta-path set in detail. As shown in Figure 1 (c), the candidate meta-path set of $e_{a_{3} p_{4}}^{t}$ includes $m_{1}:\left(a_{1}, p_{2}, c_{1}, p_{3}, a_{3}\right)$, $m_{2}:\left(a_{2}, p_{3}, a_{3}\right)$, etc. Thus, $\eta_{s}(t)=\eta_{m_{1}}(t)+\eta_{m_{2}}(t)$. Obviously, $m_{1}$ and $m_{2}$ are generated following different meta-path APCPA and APA, which dissimilarly affect different tasks. To catch this subtle distinction, we apply a semantic-level attention mechanism [Bahdanau et al., 2015]. Formally, we define the weights for different types of meta-path as follows:

$$
\omega_{b}=\frac{e^{\omega_{b}}}{\sum_{c} e^{\omega_{c}}},
$$

where $c$ is the set of all meta-path defined in the task, and $\omega_{b}$ represents the weight of $b$-th meta-path. Taking account of the semantics of different meta-path, we reformulate the influence of candidate meta-path set as

$$
\eta_{s}(t)=\sum_{t_{m}<t} \omega_{\mathcal{M}} \times \eta_{m}(t),
$$

where $\omega_{\mathcal{M}}$ is the weight of meta-path $\mathcal{M}$, and meta-path instance $m$ belongs to $\mathcal{M}$. As we defined above, $\eta_{m_{1}}(t)=$ $\eta_{e_{a_{1} p_{2}}}(t)+\eta_{e_{p_{2} c_{1}}}(t)+\eta_{e_{c_{1} p_{3}}}(t)+\eta_{e_{p_{3} a_{3}}}(t)$. Clearly, the influence of every edge should be different on account of the hop counts to training edge $e_{a_{3} p_{4}}^{t}$. Therefore, a structurallevel attention mechanism is used to capture this difference, and we denote the weights related to the hop counts as

$$
\theta_{h_{o q}}=\frac{e^{\theta_{h_{o q}}}}{\sum_{z^{\prime}} e^{\theta_{h}}},
$$

where $h_{o q}$ represents the hop counts of edge $e_{o q}$ to the source node, while the weight of $h_{o q}$-th hop is indicated as $\theta_{h_{o q}} . z^{\prime}$ is positively related to the number of candidate edges $z$. Therefore, the influence of one meta-path instance is re-defined as

$$
\eta_{m}(t)=\sum_{e_{i j} \in m} \theta_{h_{i j}} \times \eta_{e_{i j}}(t) .
$$

With the meta-path, two-level attention mechanisms, and Hawkes process, we model the evolution of every temporal edge. In this way, THINE is able to obtain multi-type node embedding with preserving semantics and structures in both static and dynamic networks. Based on the formulas above, we define the conditional intensity function $\widetilde{\lambda}_{x, y}(t)$ to represent the intensity of generating a temporal edge between nodes $v_{x}$ and $v_{y}$ at time $t$ as

$$
\begin{aligned}
\tilde{\lambda}_{x, y}(t) & =\eta_{x, y}+\eta_{s}(t) \\
& =\eta_{x, y}+\sum_{t_{m}<t}\left(\omega_{\mathcal{M}} \times \sum_{e_{i j} \in m} \theta_{h_{i j}} \times \eta_{e_{i j}}(t)\right) .
\end{aligned}
$$

Considering that the conditional intensity function should return a positive real number, an exponential function is used to transfer $\widetilde{\lambda}_{x, y}(t)$, which can be formulated as $\lambda_{x, y}(t)=$ $\exp \left(\widetilde{\lambda}_{x, y}(t)\right)$. Moreover, $\lambda_{x, y}(t)$ is squeezed between 0 and 1 simultaneously to indicate the probability of establishing a relation between nodes $v_{x}$ and $v_{y}$.

Loss function. Therefore, we can represent the probability of establishing a connection between nodes $v_{x}$ and $v_{y}$ at time $t$. Especially, with the consideration of conditional intensity function and candidate meta-path set $S(t)$ before time $t$, we 


\begin{tabular}{c|cccc}
\hline Datasets & Node Types & \# Nodes & Meta-path & Time Steps \\
\hline \multirow{3}{*}{ Aminer } & Author(A) & 10206 & APA & \\
& Paper(P) & 10457 & APPA & 10 \\
& Conference(C) & 2584 & APCPA & \\
\hline \multirow{3}{*}{ DBLP } & Author(A) & 22662 & APA & \\
& Paper(P) & 22670 & APPA & 15 \\
& Conference(C) & 2938 & APCPA & \\
\hline \multirow{6}{*}{ Yelp } & & & USU & \\
& Star(S) & 5 & BSB & \multirow{2}{*}{15} \\
& User(U) & 24586 & BUB & \\
& Business(B) & 800 & BSUSB & \\
& & & UBSBU & \\
\hline
\end{tabular}

Table 1: Data statistics

define this probability as

$$
p\left(v_{x}, v_{y} \mid S(t)\right)=\frac{\lambda_{x, y}(t)}{\sum_{y^{\prime}} \lambda_{x, y^{\prime}}(t)},
$$

where $y^{\prime}$ denotes all nodes except $v_{x}$ in temporal HIN, and the log likelihood of all node pairs can be indicated as

$$
\log L=\sum_{\left(v_{x}, v_{y}\right) \in \mathcal{E}} \log p\left(v_{x}, v_{y} \mid S(t)\right)
$$

Obviously, the calculation of $p\left(v_{x}, v_{y} \mid S(t)\right)$ needs to summarise the information of the whole network nodes. We apply negative sampling techniques [Zuo et al., 2018] to make our model more effective to mitigate huge computing overhead. So the loss function can be reformulated as

$$
-\log \sigma\left(\widetilde{\lambda}_{x, y}(t)\right)-\sum_{k=1}^{K} E_{v^{k} \sim P_{n}(v)}\left[\log \sigma\left(-\widetilde{\lambda}_{x, k}(t)\right)\right],
$$

where $K$ is the number of negative nodes sampled according to $P_{n}(v) . P_{n}(v)$ is positive correlated with $d_{v}^{3 / 4}$ while $d_{v}$ denotes the degree of node $v$. Furthermore, $\sigma$ is a sigmoid function which can be represented as $\sigma(x)=\frac{1}{1+e^{-x}}$. Finally, we optimize our model by Adaptive Moment Estimation (Adam).

\section{Experiments and Discussions}

\subsection{Experimental Setup}

Datasets. In order to demonstrate the effectiveness of THINE, we evaluate it on three real-world datasets. They are Aminer [Tang et al., 2008], DBLP ${ }^{1}$, and Yelp ${ }^{2}$, respectively. The statistics of these datasets are shown in Table 1.

Baselines. We compare THINE with several state-of-theart network embedding methods, including nine shallow and two deep embedding models, which are listed in Table 2.

Experimental settings. We evaluate THINE and other baselines on a server with Intel Xeon CPU E5-2680, Tesla V100 GPUs, and 250GB Memory. The experimental environment of software is Ubuntu 18.04 with CUDA 10.2. To be fair, the embedding dimension $\mathrm{d}$ is set as 100 for all methods. For THINE, the learning rate of Adam is set as 0.003

\footnotetext{
${ }^{1}$ https://dblp.org

${ }^{2}$ https://www.yelp.com/dataset
}

\begin{tabular}{c|c|c|c}
\hline & Methods & Heterogeneous & Temporal \\
\hline \multirow{5}{*}{ Shallow } & Deepwalk [Perozzi et al., 2014] & $\times$ & $\times$ \\
& LINE [Tang et al., 2015b] & $\times$ & $\times$ \\
& DySAT [Sankar et al., 2020] & $\times$ & $\sqrt{ }$ \\
& HTNE [Zuo et al., 2018] & $\times$ & $\sqrt{ }$ \\
& MTNE [Huang et al., 2020] & $\times$ & $\times$ \\
& Metapath2vec [Dong et al., 2017] & $\sqrt{ }$ & $\times$ \\
& StHNE [Wang et al., 2020] & $\sqrt{ }$ & $\sqrt{ }$ \\
& DHNE [Yin et al., 2019] & $\sqrt{ }$ & $\sqrt{ }$ \\
\hline \multirow{6}{*}{ Deep } & DyHNE [Wang et al., 2020] & $\sqrt{ }$ & $\times$ \\
& MAGNN [Fu et al., 2020] & $\sqrt{ }$ & $\sqrt{ }$ \\
\hline
\end{tabular}

Table 2: Baselines

while the batch size is 500. Moreover, we set the number of walks per node as 10 , the walk length is 30 , the number $n$ of candidate meta-path instances is 20 , the number $z$ of candidate edges is 4 , and the negative samples is set to be 5 . Furthermore, we use the default parameter settings for other baselines. To ensure experiments more reliable, we take the average of ten results as the final result for each experiment.

\subsection{Experiment Performance}

We first obtain node embeddings of THINE and other baselines. After that, we evaluate it by both static and dynamic tasks. For static tasks, we use two traditional measures, node classification and link prediction. Further, we use temporal link recommendation to test for dynamic effectiveness.

Node classification. We first apply all the methods to get node embeddings respectively, which are viewed as features to train a Logistic Regression classifier. Especially, the size of the training set is set as $60 \%, 80 \%$ and the remaining nodes as the test set. We use micro-f1 and macro-f1 to evaluate results.

As we can see in Table 3, THINE outperforms other methods on three datasets, which demonstrates that capturing all dynamics and the influence between different types of nodes is helpful for understanding structures of HIN. Moreover, our model has similar results when using 60 or 80 percent of the training set, which also shows the robustness of THINE.

Link prediction. We denote the representation of edge $e_{x, y}$ is $\left|u_{x}-u_{y}\right|$, where $u_{x}$ and $u_{y}$ are embeddings of nodes $v_{x}$ and $v_{y}$. We also train a Logistic Regression classifier, in which input is the representation of edges. For Aminer and DBLP, we focus on co-author relations. Therefore, we hide $25 \% A P$ randomly since co-author information is implicit in meta-path $A P A$. For Yelp, we hide $25 \%$ UR to predict the connection between users. On each dataset, we randomly choose 25,000 edges as positive ones while generating 25,000 negative ones. The results of link prediction are listed in Table 4.

We can observe that THINE outperforms all the baselines, and most dynamic methods have a good effect on link prediction, which further indicates that temporal information helps extract the structures and semantics of temporal HINs.

Temporal link recommendation. We do a temporal link recommendation experiment to test dynamic effectiveness. Especially, we train all the methods on HINs before time $t$. Then we make recommendations after time $t$. For each dataset, the first $80 \%$ of the period is used for training while 


\begin{tabular}{|c|c|c|c|c|c|c|c|c|c|c|c|c|}
\hline \multirow{3}{*}{ Methods } & \multicolumn{4}{|c|}{ Aminer } & \multicolumn{4}{|c|}{ DBLP } & \multicolumn{4}{|c|}{ Yelp } \\
\hline & \multicolumn{2}{|c|}{ micro-f1 } & \multicolumn{2}{|c|}{ macro-f1 } & \multicolumn{2}{|c|}{ micro-f1 } & \multicolumn{2}{|c|}{ macro-f1 } & \multicolumn{2}{|c|}{ micro-f1 } & \multicolumn{2}{|c|}{ macro-f1 } \\
\hline & $60 \%$ & $80 \%$ & $60 \%$ & $80 \%$ & $60 \%$ & $80 \%$ & $60 \%$ & $80 \%$ & $60 \%$ & $80 \%$ & $60 \%$ & $80 \%$ \\
\hline Deepwalk & $44.50 \%$ & $44.67 \%$ & $44.48 \%$ & $44.73 \%$ & $34.25 \%$ & $36.00 \%$ & $34.21 \%$ & $35.49 \%$ & $64.38 \%$ & $65.93 \%$ & $64.37 \%$ & $65.91 \%$ \\
\hline LINE & $36.33 \%$ & $38.33 \%$ & $34.96 \%$ & $36.21 \%$ & $29.75 \%$ & $30.38 \%$ & $29.73 \%$ & $30.36 \%$ & $56.43 \%$ & $61.75 \%$ & $55.30 \%$ & $59.73 \%$ \\
\hline DySAT & $43.00 \%$ & $42.66 \%$ & $45.00 \%$ & $44.74 \%$ & $31.50 \%$ & $32.25 \%$ & $31.26 \%$ & $32.30 \%$ & $68.44 \%$ & $69.99 \%$ & $68.37 \%$ & $69.70 \%$ \\
\hline HTNE & $37.50 \%$ & $40.83 \%$ & $35.81 \%$ & $39.08 \%$ & $30.75 \%$ & $32.63 \%$ & $30.73 \%$ & $32.68 \%$ & $61.50 \%$ & $65.00 \%$ & $61.22 \%$ & $64.75 \%$ \\
\hline MTNE & $41.33 \%$ & $45.08 \%$ & $40.51 \%$ & $45.67 \%$ & $31.25 \%$ & $33.88 \%$ & $31.04 \%$ & $33.89 \%$ & $65.00 \%$ & $68.75 \%$ & $64.99 \%$ & $68.67 \%$ \\
\hline Metapath2vec & $38.17 \%$ & $40.33 \%$ & $38.12 \%$ & $40.13 \%$ & $30.50 \%$ & $34.50 \%$ & $30.47 \%$ & $34.28 \%$ & $50.87 \%$ & $60.88 \%$ & $49.51 \%$ & $59.80 \%$ \\
\hline StHNE & $35.00 \%$ & $40.00 \%$ & $25.50 \%$ & $34.22 \%$ & $22.00 \%$ & $19.25 \%$ & $9.03 \%$ & $8.44 \%$ & $67.81 \%$ & $66.25 \%$ & $67.04 \%$ & $65.99 \%$ \\
\hline DHNE & $43.62 \%$ & $39.64 \%$ & $41.19 \%$ & $35.97 \%$ & $37.50 \%$ & $39.75 \%$ & $36.90 \%$ & $38.35 \%$ & $60.94 \%$ & $70.00 \%$ & $60.80 \%$ & $69.98 \%$ \\
\hline DyHNE & $36.33 \%$ & $42.00 \%$ & $35.30 \%$ & $41.31 \%$ & $22.13 \%$ & $19.50 \%$ & $9.06 \%$ & $8.99 \%$ & $68.44 \%$ & $68.13 \%$ & $67.74 \%$ & $67.76 \%$ \\
\hline MAGNN & $36.67 \%$ & $39.67 \%$ & $36.18 \%$ & $39.61 \%$ & $24.50 \%$ & $25.25 \%$ & $23.85 \%$ & $25.43 \%$ & $46.56 \%$ & $48.75 \%$ & $46.54 \%$ & $48.71 \%$ \\
\hline HDGNN & $41.67 \%$ & $44.83 \%$ & $41.57 \%$ & $44.61 \%$ & $37.25 \%$ & $37.50 \%$ & $36.89 \%$ & $36.85 \%$ & $62.18 \%$ & $61.87 \%$ & $62.10 \%$ & $61.80 \%$ \\
\hline THINE & $48.33 \%$ & $48.67 \%$ & $48.03 \%$ & $48.58 \%$ & $38.50 \%$ & $39.89 \%$ & $37.43 \%$ & $39.64 \%$ & $\mathbf{7 0 . 3 7 \%}$ & $71.25 \%$ & $70.23 \%$ & $71.24 \%$ \\
\hline
\end{tabular}

Table 3: Performance on node classification

\begin{tabular}{|c|c|c|c|c|c|c|c|c|c|c|c|c|c|}
\hline Datasets & Metrics & Deepwalk & LINE & DySAT & HTNE & MTNE & Metapath2vec & StHNE & DHNE & DyHNE & MAGNN & HDGNN & MTNE \\
\hline \multirow{3}{*}{ Aminer } & auc & $77.07 \%$ & $68.49 \%$ & $80.25 \%$ & $76.53 \%$ & $82.78 \%$ & $70.20 \%$ & $79.19 \%$ & $63.86 \%$ & $72.06 \%$ & $66.34 \%$ & $89.80 \%$ & $91.16 \%$ \\
\hline & f1 & $71.19 \%$ & $64.79 \%$ & $67.91 \%$ & $73.18 \%$ & $75.23 \%$ & $65.43 \%$ & $73.66 \%$ & $76.89 \%$ & $74.25 \%$ & $63.90 \%$ & $82.33 \%$ & $\mathbf{8 8 . 0 8 \%}$ \\
\hline & acc & $71.09 \%$ & $63.54 \%$ & $66.14 \%$ & $72.55 \%$ & $74.72 \%$ & $64.83 \%$ & $69.61 \%$ & $64.97 \%$ & $74.25 \%$ & $62.83 \%$ & $82.09 \%$ & $88.25 \%$ \\
\hline \multirow{3}{*}{ DBLP } & auc & $85.59 \%$ & $75.11 \%$ & $82.16 \%$ & $90.77 \%$ & $94.06 \%$ & $79.17 \%$ & $81.58 \%$ & $75.46 \%$ & $82.77 \%$ & $67.80 \%$ & $92.03 \%$ & $94.65 \%$ \\
\hline & $\mathrm{f} 1$ & $81.28 \%$ & $71.24 \%$ & $75.61 \%$ & $83.16 \%$ & $86.74 \%$ & $74.02 \%$ & $76.02 \%$ & $70.82 \%$ & $76.54 \%$ & $70.02 \%$ & $84.37 \%$ & $90.66 \%$ \\
\hline & acc & $81.28 \%$ & $70.09 \%$ & $75.03 \%$ & $82.87 \%$ & $86.61 \%$ & $73.17 \%$ & $72.47 \%$ & $69.27 \%$ & $72.97 \%$ & $63.62 \%$ & $84.17 \%$ & $90.71 \%$ \\
\hline \multirow{3}{*}{ Yelp } & auc & $50.32 \%$ & $58.30 \%$ & $53.67 \%$ & $65.27 \%$ & $67.75 \%$ & $51.18 \%$ & $73.60 \%$ & $50.41 \%$ & $75.58 \%$ & $73.29 \%$ & $76.87 \%$ & $79.33 \%$ \\
\hline & $\mathrm{f} 1$ & $63.28 \%$ & $57.56 \%$ & $54.27 \%$ & $64.77 \%$ & $65.34 \%$ & $46.99 \%$ & $70.30 \%$ & $50.52 \%$ & $71.33 \%$ & $69.19 \%$ & $71.86 \%$ & $72.36 \%$ \\
\hline & acc & $52.78 \%$ & $55.57 \%$ & $52.88 \%$ & $60.65 \%$ & $61.69 \%$ & $51.03 \%$ & $63.50 \%$ & $50.53 \%$ & $66.67 \%$ & $60.88 \%$ & $71.04 \%$ & $72.51 \%$ \\
\hline
\end{tabular}

Table 4: Performance on link prediction

remaining as a test set. Using negative squared Euclidean distance, we predict the top-5, 10 (top-2, 4) connection after time $t$ for test nodes. We then estimate results with precision and recall.

We first make recommendations between nodes of the same type since some heterogeneous baselines only obtain one type of node embedding. From Table 5, THINE has the best performance in this dynamic task. Furthermore, we can see methods for homogeneous networks generally are better than the previous HIN embedding model.

To make our results more convincing, we also make recommendations between multi-type nodes. Note that we remove some baselines or datasets that do not fit for this task. As shown in Table 6, THINE achieves the highest precision and recall among competitors. Furthermore, the methods for HIN perform better than models for homogeneous networks. This may indicate that the methods for HIN should also pay attention to the influence between nodes of the same type, which helps obtain better performance in downstream tasks.

\subsection{Parameter Analysis}

Besides, we analyze several important parameters of THINE: the number of candidate meta-path instances $n$, the number of candidate edges $z$, and the number of negative samples.

The number of candidate meta-path instances $n$. We show how the number of candidate meta-path instances $n$ influences THINE. As shown in Figure 2 (a), with the increase of candidate meta-path instances, the performance first improves and then decreases, which means that THINE does not need too little or too superfluous information from the past.
The number of candidate edges $z$. Obviously, the influence of neighbor nodes is related to the number of candidate edges $z$. From Figure 2 (b), similarly, THINE does not need too much or too little neighbor information. For a balance between complexity and performance, we set the number of candidate edges as 4 .

The number of negative samples. Finally, we test the impact of the number of negative samples on model performance. The experiment results are listed in Figure 2 (c). From the results, THINE achieves the best performance when the number of negative samples is around 5.

\section{Related Work}

Network embedding, namely graph embedding, aims to represent nodes in a low-dimensional space while preserving the properties and structures of the network. Incipiently, inspired by natural language processing [Mikolov et al., 2013], a lot of researchers focus on applying embedding on the network. Mainly, models at that time aimed to represent nodes by using the information of neighbor nodes such as Deepwalk [Perozzi et al., 2014] and LINE [Tang et al., 2015b]. However, these methods are mainly proposed for homogeneous networks and do not consider the temporal information.

Since then, network embedding goes in different directions: HIN and temporal network. For HIN, plenty of methods capture semantics and structures based on meta-path [Sun and Han, 2012] and have got powerful performance (i.e., Meta-path2vec [Dong et al., 2017], HAN [Wang et al., 2019]). Towards temporal network, some of works use matrix decomposition (DHPE [Zhu et al., 2018]), and others 


\begin{tabular}{|c|c|c|c|c|c|c|c|c|c|c|c|c|}
\hline \multirow{3}{*}{ Methods } & \multicolumn{4}{|c|}{ Aminer } & \multicolumn{4}{|c|}{ DBLP } & \multicolumn{4}{|c|}{ Yelp } \\
\hline & \multicolumn{2}{|c|}{ precision } & \multicolumn{2}{|c|}{ recall } & \multicolumn{2}{|c|}{ precision } & \multicolumn{2}{|c|}{ recall } & \multicolumn{2}{|c|}{ precision } & \multicolumn{2}{|c|}{ recall } \\
\hline & top@5 & top@10 & top@5 & top@10 & top@5 & top@10 & top@5 & top@10 & top@5 & top@10 & top@5 & top@ 10 \\
\hline Deepwalk & $9.81 \%$ & $8.03 \%$ & $2.29 \%$ & $4.50 \%$ & $9.80 \%$ & $7.98 \%$ & $2.19 \%$ & $4.25 \%$ & $0.21 \%$ & $0.34 \%$ & $0.065 \%$ & $0.120 \%$ \\
\hline LINE & $7.51 \%$ & $6.11 \%$ & $2.24 \%$ & $3.66 \%$ & $5.23 \%$ & $4.12 \%$ & $1.47 \%$ & $2.31 \%$ & $0.17 \%$ & $0.14 \%$ & $0.049 \%$ & $0.058 \%$ \\
\hline DySAT & $3.35 \%$ & $2.74 \%$ & $0.96 \%$ & $1.59 \%$ & $1.80 \%$ & $1.13 \%$ & $0.48 \%$ & $0.62 \%$ & $0.39 \%$ & $0.20 \%$ & $0.061 \%$ & $0.064 \%$ \\
\hline HTNE & $9.98 \%$ & $8.20 \%$ & $3.01 \%$ & $4.94 \%$ & $7.75 \%$ & $6.46 \%$ & $2.25 \%$ & $3.68 \%$ & $0.33 \%$ & $0.27 \%$ & $0.078 \%$ & $0.156 \%$ \\
\hline MTNE & $10.45 \%$ & $8.38 \%$ & $3.15 \%$ & $5.06 \%$ & $7.96 \%$ & $6.39 \%$ & $2.30 \%$ & $3.65 \%$ & $0.05 \%$ & $0.21 \%$ & $0.014 \%$ & $0.122 \%$ \\
\hline Metapath2vec & $2.21 \%$ & $2.46 \%$ & $0.67 \%$ & $1.49 \%$ & $2.33 \%$ & $2.07 \%$ & $0.69 \%$ & $1.23 \%$ & $0.11 \%$ & $0.21 \%$ & $0.027 \%$ & $0.122 \%$ \\
\hline StHNE & $4.60 \%$ & $3.40 \%$ & $1.40 \%$ & $2.03 \%$ & $3.67 \%$ & $2.91 \%$ & $1.01 \%$ & $1.60 \%$ & $0.39 \%$ & $0.20 \%$ & $0.064 \%$ & $0.069 \%$ \\
\hline DHNE & $3.32 \%$ & $2.24 \%$ & $1.02 \%$ & $1.34 \%$ & $6.34 \%$ & $4.87 \%$ & $1.96 \%$ & $2.91 \%$ & $0.23 \%$ & $0.11 \%$ & $0.033 \%$ & $0.033 \%$ \\
\hline DyHNE & $6.04 \%$ & $4.04 \%$ & $1.74 \%$ & $2.40 \%$ & $3.89 \%$ & $3.09 \%$ & $1.12 \%$ & $1.75 \%$ & $0.24 \%$ & $0.30 \%$ & $0.035 \%$ & $0.122 \%$ \\
\hline MAGNN & $2.65 \%$ & $2.18 \%$ & $0.81 \%$ & $1.33 \%$ & $1.62 \%$ & $1.26 \%$ & $0.45 \%$ & $0.69 \%$ & $0.32 \%$ & $0.34 \%$ & $0.055 \%$ & $0.167 \%$ \\
\hline HDGNN & $12.04 \%$ & $10.85 \%$ & $3.82 \%$ & $6.45 \%$ & $9.13 \%$ & $8.79 \%$ & $2.03 \%$ & $4.78 \%$ & $0.39 \%$ & $0.40 \%$ & $0.090 \%$ & $0.175 \%$ \\
\hline THINE & $14.05 \%$ & $12.07 \%$ & $4.31 \%$ & $7.25 \%$ & $11.67 \%$ & $9.47 \%$ & $3.48 \%$ & $5.51 \%$ & $0.40 \%$ & $0.41 \%$ & $0.105 \%$ & $0.217 \%$ \\
\hline
\end{tabular}

Table 5: Performance on temporal link recommendation between nodes of the same type

\begin{tabular}{|c|c|c|c|c|c|c|c|c|c|c|c|c|}
\hline Datasets & Metrics & top & Deepwalk & LINE & DySAT & HTNE & MTNE & Metapath2vec & DHNE & MAGNN & HDGNN & MTNE \\
\hline \multirow{4}{*}{ Aminer } & \multirow{2}{*}{ precision } & @2 & $10.33 \%$ & $6.28 \%$ & $10.46 \%$ & $8.60 \%$ & $10.46 \%$ & $17.44 \%$ & $9.43 \%$ & $8.47 \%$ & $18.30 \%$ & $22.79 \%$ \\
\hline & & @4 & $10.21 \%$ & $3.60 \%$ & $7.56 \%$ & $6.51 \%$ & $6.98 \%$ & $13.83 \%$ & $7.23 \%$ & $6.35 \%$ & $14.37 \%$ & $18.62 \%$ \\
\hline & \multirow{2}{*}{ recall } & @2 & $2.28 \%$ & $1.97 \%$ & $3.84 \%$ & $2.98 \%$ & $3.24 \%$ & $5.96 \%$ & $4.12 \%$ & $3.14 \%$ & $6.68 \%$ & $8.31 \%$ \\
\hline & & @ 4 & $6.75 \%$ & $2.21 \%$ & $5.64 \%$ & $4.61 \%$ & $4.73 \%$ & $9.76 \%$ & $5.11 \%$ & $4.23 \%$ & $10.94 \%$ & $12.91 \%$ \\
\hline \multirow{4}{*}{ DBLP } & \multirow{2}{*}{ precision } & @2 & $1.76 \%$ & $1.65 \%$ & $3.90 \%$ & $1.95 \%$ & $2.20 \%$ & $2.72 \%$ & $3.40 \%$ & $3.18 \%$ & $4.23 \%$ & $4.55 \%$ \\
\hline & & @ 4 & $2.55 \%$ & $1.02 \%$ & $3.39 \%$ & $1.29 \%$ & $1.76 \%$ & $2.57 \%$ & $3.23 \%$ & $2.97 \%$ & $3.87 \%$ & $4.08 \%$ \\
\hline & \multirow{2}{*}{ recall } & @2 & $0.54 \%$ & $0.46 \%$ & $0.96 \%$ & $0.55 \%$ & $0.64 \%$ & $0.83 \%$ & $0.84 \%$ & $0.79 \%$ & $1.26 \%$ & $1.53 \%$ \\
\hline & & @ 4 & $1.65 \%$ & $0.63 \%$ & $2.09 \%$ & $0.72 \%$ & $1.03 \%$ & $1.68 \%$ & $1.84 \%$ & $1.51 \%$ & $2.41 \%$ & $2.70 \%$ \\
\hline
\end{tabular}

Table 6: Performance on temporal link recommendation between the multi-type nodes

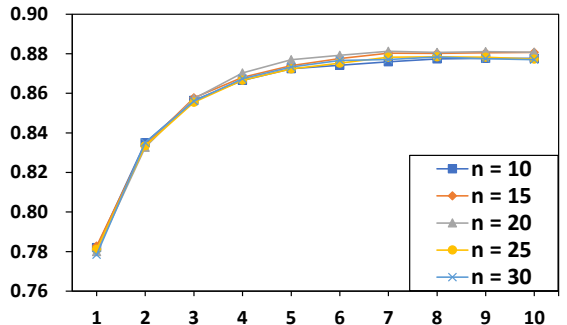

(a)

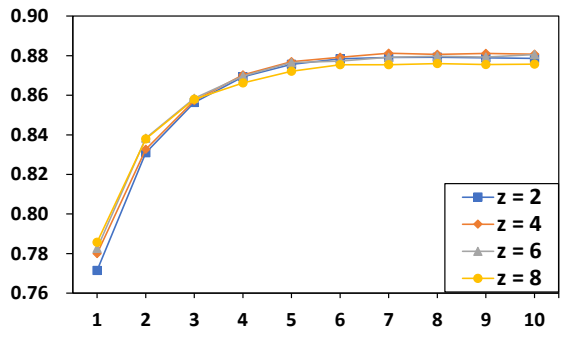

(b)

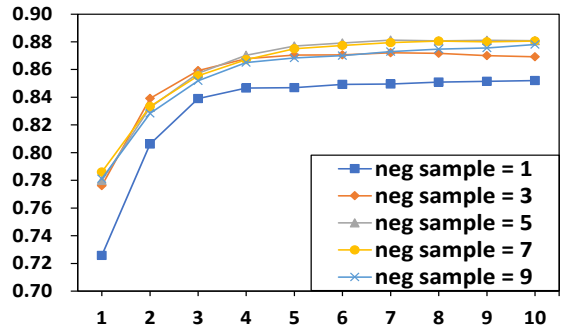

(c)

Figure 2: Experimental results of the influence with different parameters: the number of candidate meta-path instances $n$ (a), the number of candidate edges $z(\mathrm{~b})$, and negative samples (c). The X-axis is epochs, while the Y-axis represents the micro-f1 of link prediction on Aminer.

divide networks into different subgraphs of snapshots, and then aggregate the information of each subgraph, like DySAT [Sankar et al., 2020]. Fortunately, a few methods like HTNE [Zuo et al., 2018], M²DNE [Lu et al., 2019], and MTNE [Huang et al., 2020] simulate the evolution of networks.

Currently, there is an increasing trend that focuses on temporal HIN embedding. Most of such works use meta-path to capture semantics in HIN and divide time into snapshots to obtain node embeddings, like DHNE [Yin et al., 2019], Change2vec [Bian et al., 2019], and DyHNE [Wang et al., 2020]. Besides, HDGAN [Li et al., 2020] takes advantage of time-level attention mechanism to simulate network evolution. Furthermore, a few semi-supervised methods are designed for HIN, such as static method MAGNN [Fu et al., 2020] and dynamic model HDGNN [Zhou et al., 2020], but there is still a lack of deep methods for temporal HINs. More- over, none of them considers the dynamics of network evolution and multi-types node embeddings simultaneously.

\section{Conclusion}

In this paper, we propose THINE for investigating the problem of temporal HINs embedding. THINE takes advantage of the candidate meta-path set to capture structures and semantics, and uses the Hawkes process to simulate the evolution of networks simultaneously. Extensive experiments on three real-world temporal HINs show that THINE achieves the best performance in both static and dynamic tasks.

\section{Acknowledgements}

The research was supported by National Natural Science Foundation of China (No. 61802140). 


\section{References}

[Bahdanau et al., 2015] Dzmitry Bahdanau, Kyunghyun Cho, and Yoshua Bengio. Neural machine translation by jointly learning to align and translate. In Proceedings of the 3rd International Conference on Learning Representations, ICLR 2015, 2015.

[Bian et al., 2019] Ranran Bian, Yun Sing Koh, Gillian Dobbie, and Anna Divoli. Network embedding and change modeling in dynamic heterogeneous networks. In Proceedings of the 42nd International ACM SIGIR Conference on Research and Development in Information Retrieval, pages 861-864, 2019.

[Dong et al., 2017] Yuxiao Dong, Nitesh V. Chawla, and Ananthram Swami. metapath2vec: Scalable representation learning for heterogeneous networks. In Proceedings of the 23rd ACM SIGKDD International Conference on Knowledge Discovery and Data Mining, pages 135-144, 2017.

[Fu et al., 2020] Xinyu Fu, Jiani Zhang, Ziqiao Meng, and Irwin King. Magnn: Metapath aggregated graph neural network for heterogeneous graph embedding. In Proceedings of The Web Conference 2020, pages 2331-2341, 2020.

[Hawkes, 1971] Alan G. Hawkes. Spectra of some self-exciting and mutually exciting point processes. Biometrika, 58(1):83-90, 1971.

[Huang et al., 2020] Hong Huang, Zixuan Fang, Xiao Wang, Youshan Miao, and Hai Jin. Motif-preserving temporal network embedding. In Proceedings of the Twenty-Ninth International Joint Conference on Artificial Intelligence, IJCAI-20, pages 1237-1243, 2020.

[Li et al., 2020] Qiuyan Li, Yanlei Shang, Xiuquan Qiao, and Wei Dai. Heterogeneous dynamic graph attention network. In Proceedings of the 2020 IEEE International Conference on Knowledge Graph (ICKG), pages 404-411. IEEE, 2020.

[Lu et al., 2019] Yuanfu Lu, Xiao Wang, Chuan Shi, Philip S. Yu, and Yanfang Ye. Temporal network embedding with micro-and macro-dynamics. In Proceedings of the 28th ACM International Conference on Information and Knowledge Management, pages 469-478, 2019.

[Mikolov et al., 2013] Tomas Mikolov, Kai Chen, Greg Corrado, and Jeffrey Dean. Efficient estimation of word representations in vector space. In Proceedings of ICLR Workshops Track, 2013.

[Perozzi et al., 2014] Bryan Perozzi, Rami Al-Rfou, and Steven Skiena. Deepwalk: Online learning of social representations. In Proceedings of the 20th ACM SIGKDD International Conference on Knowledge Discovery and Data Mining, pages 701-710. ACM, 2014.

[Sankar et al., 2020] Aravind Sankar, Yanhong Wu, Liang Gou, Wei Zhang, and Hao Yang. Dysat: Deep neural representation learning on dynamic graphs via self-attention networks. In Proceedings of the 13th International Conference on Web Search and Data Mining, pages 519-527, 2020.
[Sun and Han, 2012] Yizhou Sun and Jiawei Han. Mining heterogeneous information networks: principles and methodologies. Synthesis Lectures on Data Mining and Knowledge Discovery, 3(2):1-159, 2012.

[Tang et al., 2008] Jie Tang, Jing Zhang, Limin Yao, Juanzi Li, Li Zhang, and Zhong Su. Arnetminer: extraction and mining of academic social networks. In Proceedings of the 14th ACM SIGKDD International Conference on Knowledge Discovery and Data Mining, pages 990-998, 2008.

[Tang et al., 2015a] Jian Tang, Meng Qu, and Qiaozhu Mei. Pte: Predictive text embedding through large-scale heterogeneous text networks. In Proceedings of the 21st ACM SIGKDD International Conference on Knowledge Discovery and Data Mining, pages 1165-1174, 2015.

[Tang et al., 2015b] Jian Tang, Meng Qu, Mingzhe Wang, Ming Zhang, Jun Yan, and Qiaozhu Mei. Line: Largescale information network embedding. In Proceedings of the 24th International Conference on World Wide Web, pages 1067-1077, 2015.

[Wang et al., 2019] Xiao Wang, Houye Ji, Chuan Shi, Bai Wang, Yanfang Ye, Peng Cui, and Philip S. Yu. Heterogeneous graph attention network. In Proceedings of The Web Conferenc 2019, pages 2022-2032, 2019.

[Wang et al., 2020] Xiao Wang, Yuanfu Lu, Chuan Shi, Ruijia Wang, Peng Cui, and Shuai Mou. Dynamic heterogeneous information network embedding with meta-path based proximity. IEEE Transactions on Knowledge and Data Engineering, 2020.

[Yin et al., 2019] Ying Yin, Li-Xin Ji, Jian-Peng Zhang, and Yu-Long Pei. Dhne: Network representation learning method for dynamic heterogeneous networks. IEEE Access, 7:134782-134792, 2019.

[Zhou et al., 2020] Fan Zhou, Xovee Xu, Ce Li, Goce Trajcevski, Ting Zhong, and Kunpeng Zhang. A heterogeneous dynamical graph neural networks approach to quantify scientific impact. arXiv preprint arXiv:2003.12042, 2020.

[Zhu et al., 2018] Dingyuan Zhu, Peng Cui, Ziwei Zhang, Jian Pei, and Wenwu Zhu. High-order proximity preserved embedding for dynamic networks. IEEE Transactions on Knowledge and Data Engineering, 30(11):2134 2144, 2018.

[Zuo et al., 2018] Yuan Zuo, Guannan Liu, Hao Lin, Jia Guo, Xiaoqian $\mathrm{Hu}$, and Junjie Wu. Embedding temporal network via neighborhood formation. In Proceedings of the 24th ACM SIGKDD International Conference on Knowledge Discovery \& Data Mining, pages 2857-2866, 2018. 